Die Revision des Betäubungsmittelgesetzes stand im Zeichen des FMH-Sessionsanlasses vom 1. Oktober 2008. Zahlreiche Parlamentarierinnen und Parlamentarier folgten der Einladung und diskutierten diese gesundheitspolitische Vorlage, über die das Schweizer Stimmvolk Ende November an der Urne entscheidet. Höhepunkt der Veranstaltung war das Referat von Dr. med. Ambros Uchten- hagen: Darin zeigte er klar und deutlich, warum das revidierte Betäubungsmittelgesetz nur befürwortet werden kann. Der Zentralvorstand der FMH ebenso wie die Delegierten haben diese Vorlage bereits vor einigen Wochen gutgeheissen.

Dr. med. Jacques de Haller, Präsident der FMH

\title{
Am eigenen Erfolg scheitern? Gute Gründe für die Revision des BetMG
}

\section{Ambros Uchtenhagen}

Präsident des Instituts für Suchtund Gesundheitsforschung, assoziiert mit der Universität Zürich

Korrespondenz:

Prof. Dr. med. et phil.

Ambros Uchtenhagen

Institut für Sucht- und

Gesundheitsforschung

Konradstrasse 32

CH-8031 Zürich

Tel. 0444481160

Fax 0444481170

isgf@isgf.uzh.ch

www.isgf.ch
Die vorgeschlagene Revision des Betäubungsmittelgesetzes (BetMG) ist überzeugend, weil sie eine Fortsetzung der Vier-Säulen-Drogenpolitik (Prävention, Therapie, Schadensminderung, Repression), die Grundlage für Aktivitäten auf Bundesebene, die Fortsetzung der heroingestützten Behandlung für therapieresistente Heroinabhängige bringt.

Vor der Implementierung dieser Politik war die Lage an der Drogenfront katastrophal: Trotz Repression bestanden ausufernde offene Drogenszenen voller Elend und negativer Auswirkungen für die Bevölkerung; trotz Prävention und Therapie nahm die Anzahl der Neueinsteiger in Heroinkonsum zu, gab es mehr Drogentodesfälle und Aidserkrankungen. Ebenso liess sich ein Anstieg drogenbedingter Kriminalität beobachten. Es bestand keine gemeinsame Handlungsgrundlage für die zuständigen Akteure und

\section{Abbildung 1}

Abnahme der Delinquenz während heroingestützter Behandlung (Quelle: Polizeidaten, $\mathrm{n}=604$; Killias et al. [1]).

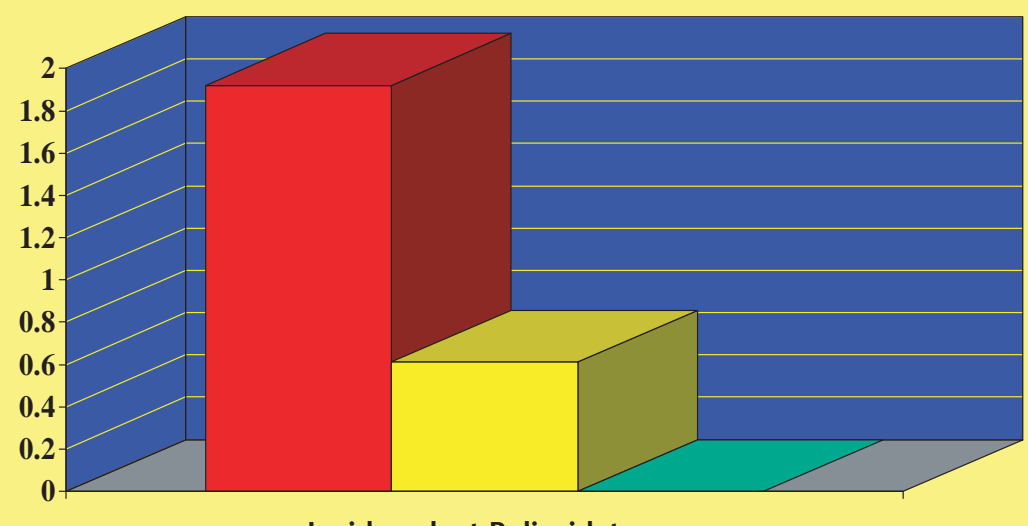

Inzidenz laut Polizeidaten

$\square$ vor Eintritt $\square$ nach 6 Mten $\square$ nach 12 Mten

auf den Ebenen Städte, Kantone, Bund. Somit gab es auch keine Aussicht auf Erfolge.

Die Vier-Säulen-Politik brachte endlich diese

Handlungsgrundlage für

- eine Zusammenarbeit der Akteure und Ebenen;

- neue Initiativen in der Prävention (z. B. Sekundärprävention bei besonders gefährdeten Zielgruppen);

- den Ausbau und die Diversifizierung aller Behandlungsoptionen sowie neue Initiativen im niederschwelligen Bereich und die Erreichung der bisher für eine Therapie nicht Erreichbaren (inkl. heroingestützte Behandlung);

- eine Neuausrichtung repressiver Massnahmen (Nulltoleranz für Drogenkonsum und -handel im öffentlichen Raum, Einrichtung von Vermittlungs- und Rückführungszentren in Zusammenarbeit Polizei-Medizin, Tolerieren nichtöffentlichen Konsums ohne Immissionen für die Bevölkerung);

- die Anerkennung und Förderung schadensmindernder Massnahmen (Aidsprävention durch Erhältlichkeit sterilen Injektionsmaterials, Injektionsräume, schadensmindernde Massnahmen im Strafvollzug).

Die Folgen dieser Politik sind u. a. ablesbar an folgenden dokumentierten Entwicklungen:

- Abnahme der Anzahl Neueinsteiger in Heroinkonsum;

- Abnahme der Drogentodesfälle;

- Abnahme der nicht in Therapie stehenden Heroinabhängigen;

- Abnahme des Risikoverhaltens und der Aidsinfektionen bei Drogenkonsumenten;

- Abnahme der drogenbedingten Delinquenz in den Städten; 
- $\quad$ keine ausufernden offenen Drogenszenen mit ihren Auswirkungen;

- weit überwiegend positive und nachhaltige gesundheitliche und soziale Folgen bei Patienten/Patientinnen in heroingestützter Behandlung.

Andererseits sind die von den Gegnern dieser neuen Politik befürchteten negativen Folgen nicht eingetreten:

\section{Abbildung 2}

Einstieg in Heroinkonsum (Inzidenzzahlen: Nordt und Stohler [2]).

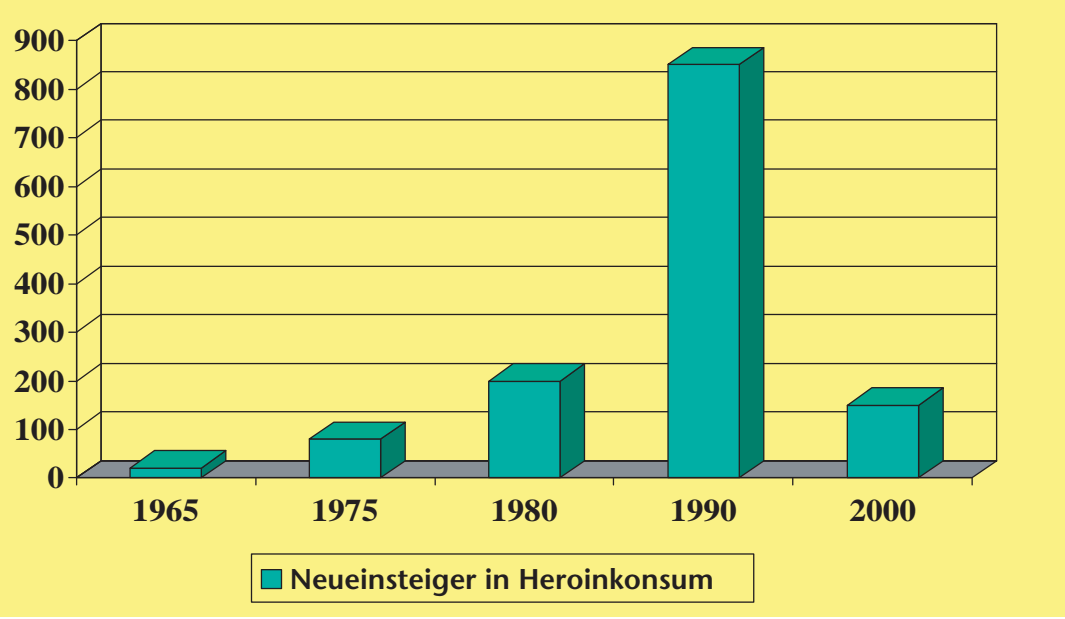

- keine erhöhte Attraktivität von Heroin für Jugendliche;

- kein Festhalten in der Heroinabhängigkeit durch heroingestützte Behandlung;

- keine Verdrängung anderer Therapieformen (u.a. abstinenzorientierter Therapien) durch die heroingestützte Behandlung.

Die Erfolge dieser Vier-Säulen-Politik sind, nach anfänglicher Skepsis, im europäischen Umfeld anerkannt und haben die Drogenpolitik und die Praxis massgeblich mitgeprägt.

Braucht es diese Politik noch? Die grundsätzliche Suchtbereitschaft in der Bevölkerung besteht nach wie vor. Derzeit stehen andere Suchtmittel als Heroin im Vordergrund (Kokain, Designerdrogen, Alkohol, Medikamente), und die Drogenprobleme sind aus dem Strassenbild und dem öffentlichen Bewusstein verschwunden. Die Weiterführung der seit 1991 eingeführten Massnahmen ist trotzdem unentbehrlich, wenn man einen Rückfall in die alten Verhältnisse verhindern will.

\section{Literatur}

1 Killias M, Aebi MF, Ribaud D. Effects of heroin prescription on police contacts among drug addicts. Eur J Crim Policy Res. 1998;6:433-8.

2 Nordt C, Stohler R. Incidence of heroin use in Zurich, Switzerland: a treatment case register analysis. Lancet. 2006;367(9525):1830-4. 